\title{
Hype or hope?
}

Microbiome research has attracted considerable attention, partially because of the potential to manipulate the microbiome for human health. To fulfil this promise, tractable methods and cautious interpretation of results are needed.

successful

translation relies on tractable methods and systems that enable systematic and controlled testing

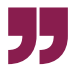

The microbiota is booming - the number of studies mentioning 'microbiome' or 'microbiota' in their title or abstract grew from 11 in 1980 to over 13,000 in 2018. Almost any human disease you can think of has proposed links with the microbiome: inflammatory bowel disease, cancer, diabetes, obesity, atherosclerosis, fatty liver disease, malnutrition, autism, Alzheimer disease, depression, autoimmunity, asthma and on goes the list. Do you want to run faster, sleep better, live longer, or have more friends? There is bound to be a claim for a 'microbiota fix'. An array of microbiome-based therapeutics are on the market or in development, ranging from prebioctics, probiotics, postbiotics, poop pills and biologics that target the gut microbiota to probiotic skin care and vaginal microbiota transplants. Furthermore, various companies offer testing of your (or your dog's) gut microbiome and then make personalized diet and lifestyle recommendations and some even sell supplements. And for the so inclined there are now even smart toilets that send faecal bacterial counts directly to your smartphone. As a microbiologist, one cannot help but think of some of these developments as the Wild West of microbiome science.

However, it is clear that we live in close association with our microbiota and their functions are manifold. Furthermore, many human diseases are complex and for some, current treatments or preventative measures are insufficient and targeting the microbiota could be a potential way to deal with some of these challenges. The prime example is Clostridioides difficile infection, which can be efficiently treated with faecal microbiota transplantation. Owing to technological and analytical advances, our knowledge of microbiota diversity and functions and their roles in disease is rapidly increasing, and efforts are under way to go beyond disease associations and to translate this knowledge into therapeutics and applications. In large part, successful translation relies on tractable methods and systems that enable systematic and controlled testing of hypotheses and interventions. To highlight the latest developments in microbiome tractability and translation, we have put together a special Focus issue on these topics.

Models are essential tools to understand disease mechanisms and the specific molecular links that determine host-microbiota associations and interactions. In recent years, microbiome research has increasingly expanded to the use of simple animal models such as flies, zebrafish and worms. Angela Douglas argues that these simple models are cost-effective and time-efficient, and are particularly useful for large screens or multivariate experimental designs.

Once such a screen has identified a trait or relationship to target, microbiome engineering can be used to develop interventions. Chris Lawson, Trina McMahon and colleagues detail the design-build-test-learn cycle to efficiently engineer microbiomes for various applications. This approach has been successfully used in other fields such as metabolic engineering and provides a tractable path for microbiome translation.

One potential application is manipulating the human gut microbiota to treat or prevent diseases such as diabetes mellitus or cardiovascular disease. There is a clear link between nutrition, the gut microbiome and health. However, large individual differences make it difficult to predict the direction and strength of responses and thus to therapeutically manipulate this link. Eran Elinav and colleagues discuss the considerable variation in how individuals and their microbiome react to diet and how personalisation could optimize any therapeutic or preventative interventions that target this axis.

The human gut is a complex environment and Jeroen Raes and colleagues argue that a systems ecology perspective is needed to understand the gut microbiota. They provide an outline of how culturing and in vitro interaction experiments, combined with computer modelling, can help us to develop a functional and mechanistic understanding of the gut microbiota, which is the basis for successful translational projects.

In summary, advances in modelling, be it animal, in vitro or in vivo models, and ever increasing datasets raise great hope for microbiota translation and applications. In this context, a little bit of hype might not only have negative consequences. After all, the strong interest in microbiome science has led to funding initiatives and industry engagement (as reflected by predicted growth rates of the microbiome market of over $20 \%$ per year). It is time to further strengthen the scientific basis from which microbiome translation can grow. 